\title{
La experiencia de la inclusión de arte efímero en Madrid a través de las instituciones regladas. Madrid Abierto, 2004-2011
}

\author{
LUCILA URDA PEÑA \\ Arquitecto. Profesora asociada Escuela Técnica Superior de Arquitectura \\ Universidad Politécnica de Madrid \\ lucila.urda@upm.es
}

\begin{abstract}
Resumen
La ciudad de Madrid ha experimentado en los últimos siete años pequeñas transformaciones temporales a través de la inserción de actuaciones artísticas urbanas en paralelo a la feria de arte contemporáneo ARCO. A raíz de esta feria surge una interesante iniciativa consistente en trasladar a la calle aquellas reflexiones artísticas más actuales. Comenzando su ubicación en un contexto físico vinculado directamente con la feria se fue trasladando, en principio, a calles representativas de la ciudad de Madrid y desplazándose, a medida que evolucionaba Madrid Abierto hacia barrios menos céntricos. La voluntad de hacer más accesible el arte para los ciudadanos así como la búsqueda de la interacción ciudad-arte fue desencadenando a lo largo de los años la aparición de proyectos cada vez más involucrados con el espacio físico y social de Madrid. La tendencia de la evolución del planteamiento de los proyectos artísticos estaba dirigida claramente hacia la intermediación del artista como agente catalizador de la relación del ciudadano con su ciudad. Las intervenciones pasaron de ser puramente artísticas a tratar de servir como lugar de encuentro y reflexión sobre situaciones urbanas concretas, planteando de una manera más clara la participación del vecino en los proyectos a través del estudio, en algunos casos, de necesidades reales en barrios determinados. El interés de la evolución de los proyectos radica en la posible continuidad de las actuaciones, planteadas, en principio, como efímeras, al tratar de trabajar con el espacio social simultáneamente al espacio físico de los lugares elegidos. La valoración del efecto real de las intervenciones de los últimos años nos plantea la hipótesis de la posibilidad del arte como transformador del espacio público, entendido este como lugar de encuentro y pensamiento colectivo.
\end{abstract}

Palabras clave: Madrid Abierto. 


\title{
The experience of the inclusion of ephemeral art in Madrid through regulated institutions. Madrid Abierto, 2004-2011
}

\begin{abstract}
The city of Madrid has experienced in the last seven years small temporary transformations through the inclusion of urban artistic performances in parallel to contemporary art fair ARCO. As a result of the art fair ARCO arises an interesting initiative consisting in moving to the street those more current art reflections. Starting its location in a physical context directly linked to the fair it was moving, in principle, from representative streets of the city of Madrid towards less centrally located neighborhoods. Willing to do more accessible art for citizens as well as the search for the city-art interaction was triggering the emergence of projects increasingly involved with the physical and social space of Madrid over the years. The trend of the evolution of the approach to the artistic projects was clearly directed towards the intermediation of the artist as a catalyst of the relationship of the citizen with his city. The interventions moved from being purely artistic to serve as a place of encounter and reflection on specific urban situations, posing in a clearer way the participation of the neighbor in the projects through the study, in some cases, actual needs in certain neighborhoods. The interest of the evolution of the projects lies in possible continuity of actions, raised, in principle, such as mayflies, trying to work with the social space simultaneously to the physical space of the chosen places. The valuation of the real effect of the interventions of the past few years presents us the hypothesis of the possibility of art as a transformer of the public space, understood as a place of encounter and collective thinking.
\end{abstract}

Key words: Madrid Abierto.

Madrid Abierto surge como una iniciativa al abrigo de la feria internacional de Arte Contemporáneo ARCO que continúa celebrándose anualmente en el recinto Ferial IFEMA de Madrid. En sus comienzos Madrid Abierto aparece ubicada físicamente en el mismo lugar que ARCO, en los llamados open spaces que habilita en el año 2000 la propia feria con el patrocinio de la Fundación Altadis, fundación dependiente de la empresa franco-española del mismo nombre que en 2008 fue adquirida por la empresa inglesa Tobacco. En los Open spaces se exponían obras de artistas tanto en el espacio público próximo a la feria como en el espacio expositivo interior. Se instalaron, por 
tanto, esculturas en los espacios de acceso de la feria y se organizaron mesas de debate sobre el espacio público. En el año 2001, tratando de impulsar el proyecto se constituye un jurado presidido por Jérôme Sans, codirector entonces del Palais de Tokio y se premian las mejores obras. Sin embargo esta experiencia poco tenía que ver con "arte público" y, por tanto, fue muy criticada por distintos artistas, colectivos e instituciones culturales.

En este contexto se trata de buscar la manera de hacer real la experiencia del arte en el espacio público más allá de la inserción de ciertas esculturas en determinadas zonas de paso. De la reflexión de los responsables de ARCO y aquellos de la fundación Altadis surge la idea de la inserción de arte efímero en las calles de Madrid.

Las intervenciones a partir de ese momento se enmarcan en la definición de Lucy R. Lippard de arte público como cualquier tipo de obra de libre acceso que se preocupa, desafía, implica, y tiene en cuenta la opinión del público para quien o con quien ha sido realizada, respetando a la comunidad y al medio. El resto es obra privada, no importa lo grande o expuesta o molesta que sea, o lo muy de moda que esté (Lippard, 2001: 61). La iniciativa chocaba con las políticas municipales precedentes en Madrid que limitaban el uso del arte a la inserción de determinadas esculturas de corte neorrealista que representaban un supuesto imaginario colectivo retrógrado y de referentes toscos e inmediatos como la famosa Violetera de la Gran Vía.

Es en estos momentos cuando se encarga el comisariado a Jorge Díez con objetos de definir los objetivos y las estrategias de Madrid Abierto. Para ello se estudian otros casos de ciudades con experiencias similares tanto en el ámbito nacional como en el ámbito internacional. A nivel internacional se estudian el Skulptur Projekte de Münster (Alemania), cuya primer edición se celebró en 1977, o inSite_05 en la frontera entre Estados Unidos y México, en San Diego-Tijuana, que se desarrolló entre 2003 y 2005 con el objetivo de estimular experiencias de dominio público en el interior de los flujos cotidianos de dicha zona fronteriza, además de otras iniciativas como la Muestra de luces de artistas asociada a la promoción de los Juegos Olímpicos de Invierno de Turín o el programa de arte público paralelo a la Bienal de Estambul (Turquía), salvando las distancias en cuanto a su escala o temática.

Otras experiencias nacionales interesantes también fueron referencia para los iniciadores de Madrid Abierto. Se estudiaron aquellas que basan sus re- 
flexiones en el contexto social y político, generalmente desde posiciones críticas, para interactuar con esos campos implicando al público. Una de estas iniciativas fue Capital Confort, desarrollada por el colectivo de artistas El Perro en Alcorcón (Madrid) entre 1997 y 2002 e Idensitat ${ }^{1}$, denominada Calaf Arte Público en su primera edición en 1999 y dirigida por Ramón Parramón, que comienza con un concurso de escultura de Calaf (3.435 habitantes) de la provincia de Barcelona, y se extiende a lo largo del tiempo a otras ciudades como Mataró o Manresa. Tanto Ramón Parramón como El Perro reconvertido en el colectivo Democracia participaron posteriormente en distintas ediciones de Madrid Abierto como comisarios o colaboradores. La financiación en un primer momento se limita el presupuesto proporcionado por la fundación Altadis en la primera edición, la colaboración del Ayuntamiento y de la Comunidad de Madrid será progresivamente mayor desde el punto de vista económico y de gestión a lo largo de los años de las ediciones de Madrid Abierto.

Con la incorporación de Jorge Diez en 2003 para afrontar el liderazgo de Madrid Abierto se propone una gestión que permita destinar casi la totalidad del presupuesto a los distintos proyectos propuestos por los artistas que, en el primer año, pasan de ser escogidos entre los artistas emergentes del momento a, en 2004, ser seleccionados a través de la convocatoria de un concurso público internacional. ARCO cedió un espacio dentro de la feria para presentar Madrid Abierto, difundió la convocatoria internacional y participó en la producción de una de las intervenciones. La financiación inicial corrió a cargo de Altadis, Ayuntamiento y Comunidad de Madrid, que asumieron de forma directa los distintos gastos del proyecto, y se gestionaron patrocinios con instituciones culturales radicadas en el eje primer de actuación (Paseo de Recoletos), como Casa de América, Círculo de Bellas Artes o Fundación Canal, y con entidades vinculadas al arte contemporáneo como, por ejemplo la Fundación Telefónica. Además el progra-

${ }^{1}$ ID \# 1 IDENSITAT se inicia en Calaf en 1999 como un proyecto que desarrolla intervenciones en el espacio público mediante propuestas de creadores procedentes de diferentes disciplinas. A partir de una convocatoria pública internacional se seleccionan proyectos que pueden incidir en dinámicas sociales y que aportan investigación y experimentación a partir de un trabajo de campo previo. IDENSITAT entiende la creación como un proceso de trabajo vinculado a un espacio, a un contexto concreto, proponiendo mecanismos de implicación en el ámbito social. http://www.idensitat.net /index.php?option=com_content\&view=category\&layout=blog\&id=9\&Itemid=15. 
ma Metrópolis de La2 de TVE produjo un programa monográfico sobre la primera edición y que la Sociedad Estatal para el desarrollo del Diseño y la Innovación (Ddi) se hizo cargo del espacio promocional e informativo en ARCO.

La gestión se fue desarrollando con éxito, sin embargo, los cuatro proyectos de la convocatoria de 2003 quedaron bloqueados por el Ayuntamiento bajo el mandato de Álvarez del Manzano. En 2004 se lanza el concurso internacional y se incorporan los proyectos que, finalmente, no se pudieron llevar a cabo el año anterior. Las intervenciones se desarrollan entre el 5 y el 22 de febrero de 2004. La convocatoria fue un éxito (234 propuestas de 316 artistas de 25 países) y los patrocinadores quedaron satisfechos, por lo que se decide continuar. A partir de 2005 las convocatorias siguen la estructura de la primera, aunque el espacio de actuación en torno al Paseo de Recoletos se va ampliando. En este mismo año las fachadas de la Casa de América (Plaza de Cibeles) y del Círculo de Bellas Artes son intervenidas (Calle Alcalá). Se interviene también en algunas paradas de autobuses y los propios vehículos a lo largo del eje Castellana-Recoletos-Prado ${ }^{2}$. Se utilizan además elementos presentes en el paisaje urbano de la ciudad para intervenir en ellos como los mupis publicitarios ${ }^{3}$ de las paradas de autobuses y se usa la infraestructura de la red "canal metro" para transmitir proyectos concretos y, por tanto, las intervenciones trascienden al acotado espacio original.

A partir de las primeras experiencias, casi todas ubicadas en el eje Castellana-Prado-Recoletos o espacios representativos aledaños (calle Alcalá, Gran Vía, Plaza de Colón) en 2008 se decide modificar el planteamiento original tanto en su planteamiento como en la ubicación de las propuestas, para ello se toman las siguientes medidas:

- Mantener la convocatoria abierta con un máximo de un 50\% más de artistas invitados por los comisarios.

- Establecer una periodicidad bienal, dedicando el primer año a las mesas de debate, incluida la presentación de los artistas seleccionados, e

\footnotetext{
${ }^{2}$ Espacio móvil. Compañía de Caracas (intervención sobre los autobuses de las líneas 14 y 45 : Castellana-Recoletos-Prado).

${ }^{3}$ R. Chaves “El río, las cosas que pasan”. Proyecciones en canal metro y mobiliario urbano.
} 
incorporando las sesiones de trabajo conjunto con éstos en un formato de taller abierto. Continuar realizando las intervenciones en el mes de febrero del segundo año.

- Incluir en la convocatoria una breve introducción que contextualice de forma general la convocatoria más un texto específico de los comisarios.

- Proseguir el desbordamiento físico y conceptual del eje PradoRecoletos-Castellana.

- Continuar con las secciones de audiovisual y sonoro y ampliar los medios específicos para su emisión.

- Mantener la comisión asesora actual hasta completar un ciclo en el que todos sus miembros hayan desarrollado tareas de comisariado en Madrid Abierto.

- Generar documentos y publicaciones que desarrollen la experiencia y el conocimiento acumulado en las ediciones realizadas.

Por tanto una de las líneas de actuación plantea claramente el exceder los límites del principal eje físico de intervención que se había utilizado hasta el momento. A partir de ese momento se revisa el planteamiento de Madrid Abierto para establecer un vínculo más real del arte con la ciudad que excediera de las acciones más puramente físicas para constituir una conexión con la sociedad madrileña representada por determinados barrios. Para ello se decide reflexionar junto a los artistas seleccionados durante el proceso de generación y concreción de sus obras a partir de encuentros y debates con colectivos madrileños o extranjeros en los que la problemática social es un pilar importante de su trabajo.

En febrero de 2009 se realizó en La Casa Encendida de Madrid el seminario Urban Buddy Scheme ${ }^{4}$, junto con la presentación de los artistas y el encuentro preparatorio de sus proyectos bajo el formato de taller abierto. Como representación de colectivos españoles acudieron Basurama, C.A.S.I.T.A., Javier Duero, Exprimentolimon, Uriel Fogué, Andrés Jaque, Kawamura-Ganjavian, Ludotek, Luis Úrculo y Wunderkammer, y de otros países Alexander Gerdel (Venezuela), Internacional Festival (Suecia), Kyong Park (EE.UU), Adriana Salazar (Colombia) y STEAL TH. unlimited (Serbia/Países Bajos).

${ }^{4}$ http://madridabierto.com/es/noticias/2009/urban-buddy-scheme-seminario-y-encuentro.html 
El seminario sirvió tanto para conocer e intercambiar experiencias y para reflexionar sobre los procesos y resultados de los trabajos de colaboración socioculturales y políticamente comprometidos. Se trataba también de valorar en qué medida los procesos creativos en este contexto sirven de catalizadores para incentivar transformaciones concretas en determinados ámbitos de la ciudad. Debido al nuevo formato bienal el trabajo de los artistas del primer año se dedicó casi exclusivamente al conocimiento de la ciudad y el trabajo sobre barrios más concretos, indagando tanto en los aspectos más físicos como en su expresión social a través de los agentes sociales más locales.

La propuesta de la última edición de Madrid Abierto, tal y como comentaba Cecilia Anderson ${ }^{5}$ es abrir los procesos artísticos a la participación ciudadana para establecer conexiones con las comunidades urbanas a través del conocimiento de sus necesidades y problemáticas.

La finalidad de un enfoque tan comprometido con la sociedad es crear una red más amplia que, idealmente, pueda acercarnos a comprender algunas de las dinámicas que actúan en la ciudad. La expresión de preocupaciones concretas y la manifestación de las cuestiones locales se sitúan un paso más cerca de la resolución de un conflicto o de la mejora de una situación. (Díez, 2008/2012).

A partir de este planteamiento las actuaciones de la última edición de Madrid Abierto se constataron en los siguientes proyectos:

\section{Infomab. Kawamura Ganjavian.}

INFOMAB2010 es a la vez un lugar de tránsito y de estancia. Alberga pequeños eventos y también es un lugar desde el que repartir información a los peatones del concurrido boulevard del Paseo de Recoletos. El pabellón es un depósito cilíndrico industrial al que se le han realizado dos grandes perforaciones (accesos) y múltiples pequeñas aberturas (ojos de buey). Su presencia, a medio camino entre el ready-made y la instalación urbana, pretende darle una imagen robusta y a la vez acogedora. Se instala el puesto de información en el bulevar peatonal central de lado este del Paseo de Recoletos. La presencia de este objeto inusual tanto

${ }^{5} \mathrm{http}: / /$ madridabierto.com/es/textos/2009/introduccion.html 
por su aspecto como por su ubicación invita al transeúnte a acercarse e informarse del porqué de su presencia. La información sobre Madrid Abierto abre nuevas rutas y alternativas al paseo habitual por las calles de Madrid.

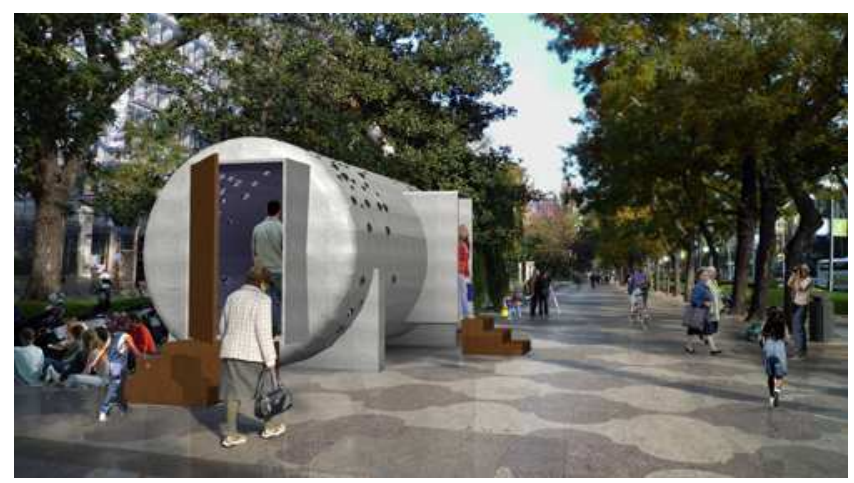

Fig. 1. INFOMAB. Pabellón para la información de Madrid Abierto. Diseño: Kawamura Ganjavian.

\section{Ghostown. Laurence Bonvin.}

Ghostown es un proyecto fotográfico centrado en algunos de los nuevos barrios de Madrid y sus alrededores, principalmente Seseña -en el sur- y Valdeluz al norte-. La serie recoge el estado de estos lugares tras la crisis financiera que ha afectado severamente a España a finales de 2008, desencadenando el colapso del sector inmobiliario. Así, Ghostown es un viaje a través de un extraño vacío y el melancólico estado sin vida en que han quedado estas áreas de construcción siempre en desarrollo. Se mostró en varios lugares: el diario Público, en el Infomab y en el Espacio 28004.

\section{Torre. Pablo Valbuena.}

La pieza consiste en una instalación específica donde se superpone en un elemento su propia representación abstracta. Con el dibujo de un plano sobre la arquitectura de referencia, se extiende la ciudad real hacia algo virtual. El medio utilizado es la videoproyección pero más allá de lo técnico, el trabajo se basa en herramientas tan antiguas como la perspectiva o el trampantojo que funcionan manipulando la percepción del espectador. La transformación del espacio urbano se logra gracias al movimiento, a la luz/ sombra. En lugar de construir arquitecturas con materiales físicos, es la proyección de líneas y planos de luz, los que generan la ilusión de nuevos espacios. 


\section{Lara Almarcegui. Bajar al subterráneo recién excavado.}

Lara Almarcegui participó, en la construcción de un parking bajo la calle Serrano. El único día a la semana en que los trabajadores no excavan se organizaron visitas guiadas al mundo subterráneo de Madrid. Esta parte suburbana de Madrid, sus entrañas, sus estratos desnudados en capas, presenta una parte asombrosa de una ciudad que de otro modo sólo se podría apreciar por su entorno ordenadamente construido. El proyecto tiene que ver con conocer la realidad física de Madrid, roca-tierra, antes de ser ciudad. Además, en este descenso al subterráneo, Almarcegui busca que el visitante experimente el subconsciente negativo de la ciudad. Es decir, en lugar de construir, bajar para saber más, pero también para hacer simple y brutalmente lo contrario que hace la arquitectura: construir con el vacío.

\section{Adaptive actions. Campo AA.}

En un espacio público de Madrid, la estación de Atocha, un Campo de producción para la acción en vivo será sede del proyecto Adaptive Actions durante casi un mes. Experiencias y materiales (ya pertenezcan a esta producción o a producciones pasadas o futuras) serán presentados en el Campo y en la subsiguiente publicación. Los visitantes tendrán la posibilidad de modificarlos o enviar sus propias propuestas. El personal de apoyo ayudará a los participantes y debatirán posibles añadidos, acciones y consiguientes asuntos con ellos. El programa de eventos en el Campo AA-Madrid (talleres/acciones, almuerzos para el aprendizaje) centrado en temas y localizaciones específicos, pretendía crear vínculos entre los participantes y las acciones con un proyecto artístico común. Se trataba de enriquecer el concepto de comunidad a través de estas diferencias.

\section{Teddy Cruz, M7Red, Iago Carro. Vallecas, ¿cómo nos van a ayudar con su arte?}

La Fachada de la Casa de América como sitio de producción. Para Madrid Abierto los artistas extendieron esta investigación para transportarla al contexto de Madrid y la fachada de la Casa de América. Mas allá de una intervención puramente visual, su propuesta tiene que ver con generar triangulaciones entre instituciones, redes y actores sociales, recursos económicos y un barrio específico de Madrid. La Fachada de la Casa de América como sitio de producción. El texto contextualiza emblemáticamente la intervención al apli- 
car en la fachada una pregunta que un inmigrante paraguayo expresó a un grupo de artistas durante un dialogo en Villa 31, un asentamiento informal dentro de Buenos Aires, en el que Teddy Cruz y M7Red colaboraron.

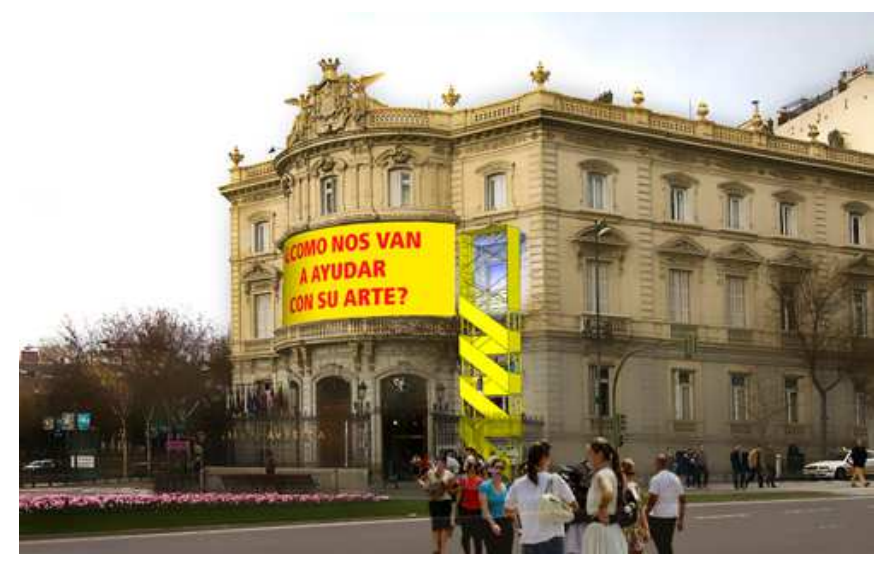

Fig. 2. Vallecas ¿cómo nos van a ayudar con su arte? Croquis previo a la intervención. Fuente: Teddy Cruz, M7Red, Iago Carro.

\subsection{Iñaqui Larrimbe. Unofficial tourism.}

El proyecto Unofficial tourism se definió, desde la economía de medios -el 'háztelo tu mismo'- dentro de los mecanismos creados por las industrias de la cultura y del turismo. No deja de ser ésta una labor de parasitismo, de habilitar estructuras paralelas a las oficiales haciendo uso de espacios e infraestructuras públicas para poder generar así otras narrativas sobre la ciudad de Madrid. Se trató de trasladar la idea de 'fanzine' al terreno de la difusión turística, creando una "oficina underground" con recursos que puedan estar al alcance de cualquier ciudadano: una caravana, principalmente. Unofficial tourism trató de ser un proyecto en respuesta y para atender a la demanda de entretenimiento cultural que las nuevas políticas culturales cimentadas en criterios socioeconómicos han generado.

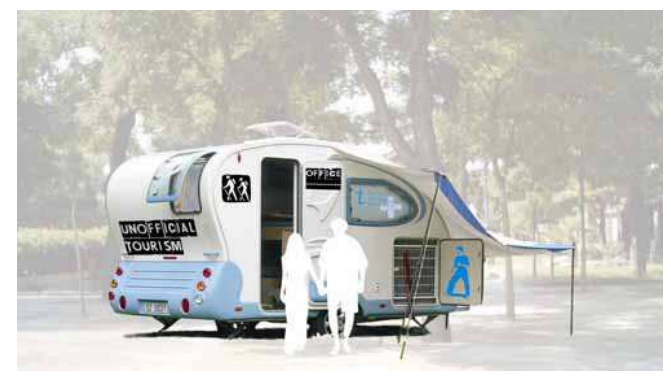

434
Fig. 3. Unofficial tourism. Fotomontaje de la caravana informativa de las rutas.

Fuente: Iñaqui Larrimbe. 


\section{Gustavo Romano. Time Notes.}

La propuesta para Madrid Abierto consistió en la apertura de una Oficina Móvil y una Oficina Central ubicada en la Casa de América. La Oficina Móvil se desplazó diariamente, desde su base en la Casa de América, por tres recorridos principales: línea Norte, tomando el Paseo de Recoletos en dirección hacia el Paseo de la Castellana; la línea Sur, por el Paseo del Prado hacia la estación de Atocha, y la línea Oeste, que tomó la calle Alcalá hacia la Plaza Mayor, derivando eventualmente por calles aledañas y desviándose de las arterias principales. El proyecto Time Notes aglutinó una serie de acciones en espacios públicos utilizando un nuevo sistema de dinero basado en unidades temporales: billetes de 1 año, 60 minutos, etc., y en cuyo

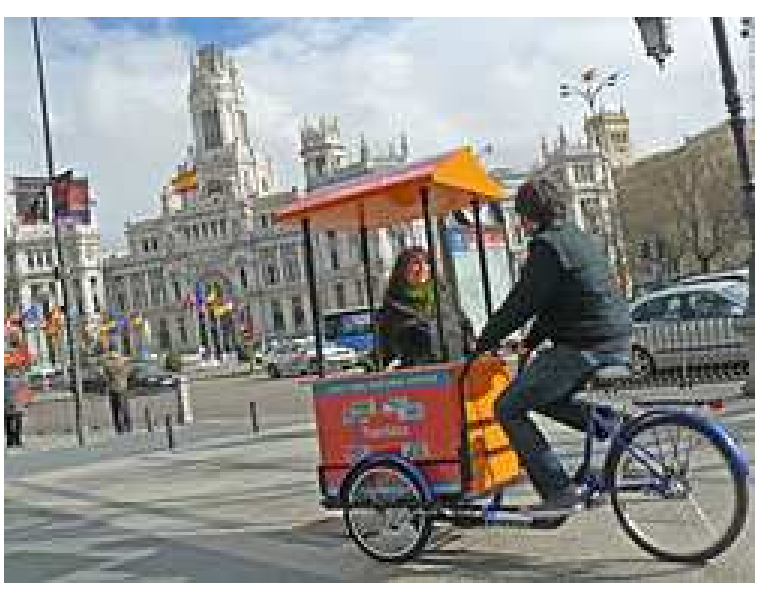

Fig. 4. Time Notes. Fotografía durante la intervención en la Plaza de Cibeles. Fuente: Gustavo Romano

reverso pueden leerse diversas citas de filósofos, escritores o economistas acerca del tiempo o del dinero. (Tiempo perdido y préstamo del tiempo).

\section{Lisa Cheung. Huert-o-bus.}

Huert-o-Bus fue un invernadero viajero, invitando a los residentes de Madrid a que cultiven verduras frescas y plantas en el centro de la ciudad. Huert-o-Bus intenta hacer uso de los espacios públicos urbanos existentes para crear jardines comunales y permitir el crecimiento local de alimentos. Como un autobús público de la ciudad, Huert-o-Bus parará por diversos barrios y distritos, en plazas señaladas, con sus 'pasajeros' de semillas y plantas pequeñas. Cada sitio estará marcado por una 'parada de autobús' que informe a vecinos/visitantes del horario y las actividades del Huert-o-Bus. Huert-o-Bus recuerda las tradiciones de asignaciones de terreno en el Reino Unido y otros países europeos. La actuación se hace en cinco distintos barrios de Madrid. 


\section{Susanne Bosch. La hucha de los deseos.}

El proyecto de arte público Hucha de deseos: ¡Todos somos un barrio, movilizate! trató de recolectar la mayor cantidad de estas pequeñas potencialidades individuales que sea posible. La artista Susanne Bosch las acumula para convertirlas en cosas tangibles, proyectos y resultados en el mundo real. El proyecto consistió en ubicar una hucha gigante para recaudar pesetas en el barrio. El montante de esa colecta se gastó en aquello que los ciudadanos decidieron que era lo más necesario en el barrio después de muchas reuniones: árboles. El proyecto sirvió también para crear vínculos y generar un sentimiento de ciudadanía y comunidad al encontrar afinidades en un proyecto conjunto.

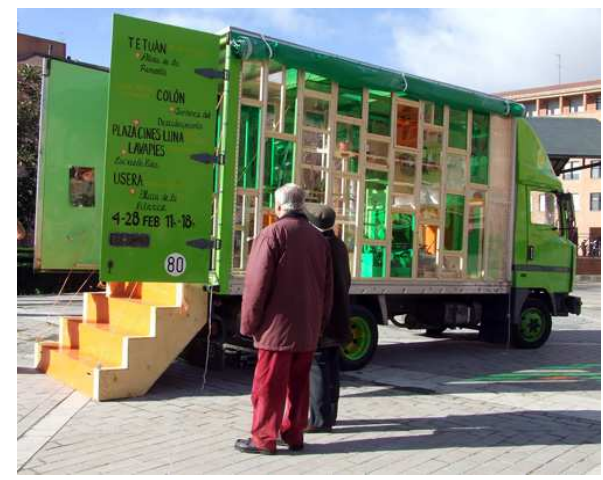

Fig. 5. Huert-o-Bus. Fotografía durante la intervención en la Plaza de la Remonta. Fuente: Lisa Cheung.

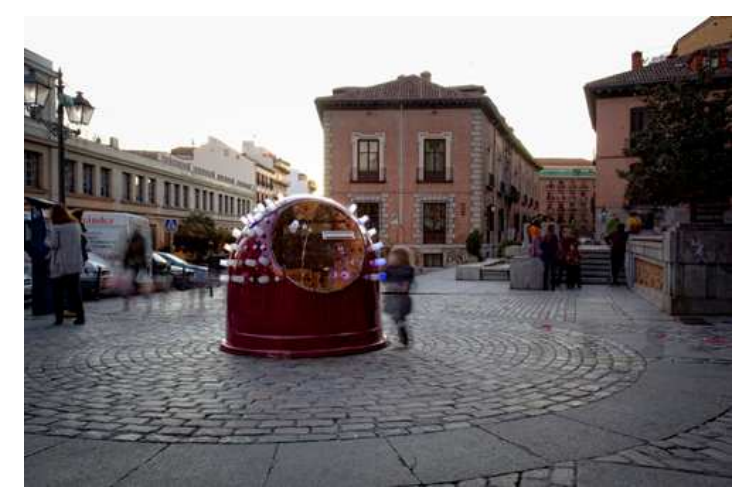

Fig. 6. La hucha de los deseos. Fotografía durante la intervención en la Plaza de los Carros. Fuente: Susanne Bosch.

El mapa de intervenciones de Madrid Abierto fue desplazándose desde el recinto ferial hacia el centro de la ciudad para terminar sobrepasando el área más representativa de la ciudad y explorar otros barrios.

Con el conocimiento de otras problemáticas y el estudio más profundo de situaciones urbanas se produce un doble efecto: colonización de más partes de ciudad y visibilización de dinámicas urbanas existentes directamente relacionadas con barrios de Madrid.

En las últimas ediciones cambia la temática de los artistas y la vida de ciertos barrios se ve definitivamente alterada a causa de determinadas accio- 
nes. Este proceso evolutivo se ve bruscamente cercenado por la falta de financiación desde 2010 hasta nuestros días.

Madrid Abierto como nació desapareció y ahora sólo existe como espacio de debate y reflexión artística y urbana. De momento sólo constan las publicaciones de todas las ediciones y hasta el momento se ha perdido la posibilidad de continuar mostrándose como escaparate de las últimas tendencias artísticas urbanas.

Esperamos que el éxito de las ediciones así como el camino que iba tomando hacia transformarse en un recurso para la catarsis social permita recuperarlo en un futuro.

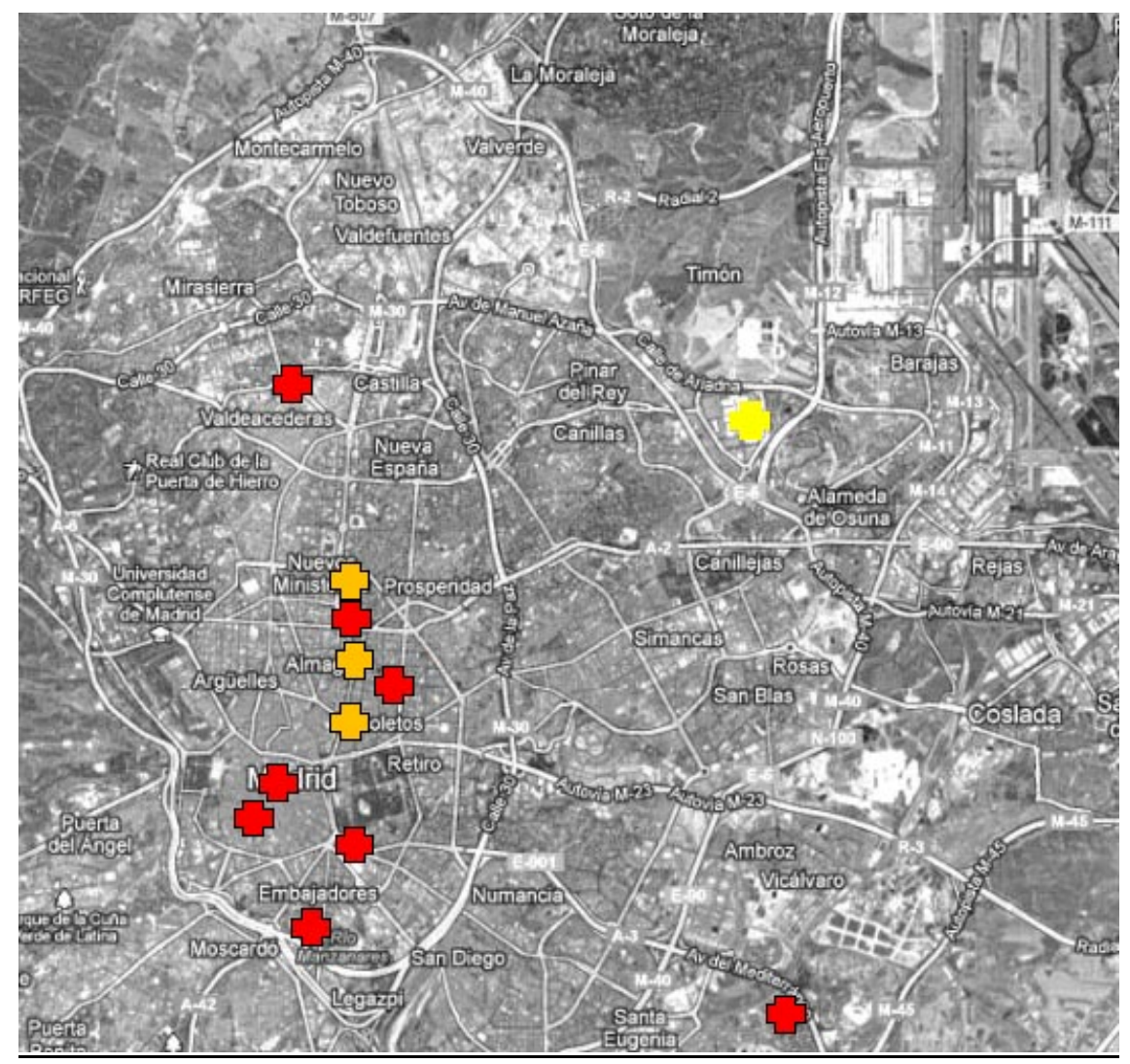

Fig. 7. Localización de intervenciones de Madrid Abierto En amarillo 2002, naranja 2004-2008 y rojo 2009-2011. 


\section{Bibliografía y referencias web.}

DíEZ, J. (2008): Madrid Abierto 2004-2008, Asociación cultural Madrid Abierto.

DíEZ, J. (2012): Madrid Abierto 2008-2012, Asociación cultural Madrid Abierto. LIPPARD, Lucy R. (2001): “Mirando alrededor: dónde estamos y dónde podríamos estar". Modos de hacer: Arte crítico, esfera pública y acción directa. Universidad, Salamanca.

http://www.madridabierto.com/

http://www.studio-kg.com/

http://www.pablovalbuena.com/

http://www.laurencebonvin.com/splash.html

http://www.adaptiveactions.net/

http://estudioteddycruz.com/

http://www.gustavoromano.org/

http://www.lisacheung.com/

http://www.susannebosch.de/ 